\title{
Cuestionario de bruxismo autoinformado. Estudio piloto en el noreste de México ${ }^{1}$
}

\section{Self-reported bruxism questionnaire. A pilot study in northeastern Mexico}

\begin{abstract}
Norma Cruz Fierro², Mónica T. González Ramírez ${ }^{3}$ y Minerva T. Juno Vanegas Farfano ${ }^{4}$
${ }^{1}$ El estudio fue aprobado por el comité de bioética de la Facultad de Odontología de la Universidad Autónoma de Nuevo León (SPSI-010613. 0046).

${ }^{2} \mathrm{PhD}$. Cirujano Dentista, Especialidad y Maestría en Odontología Restauradora. Doctora en Filosofía con orientación en Psicología. Profesor de tiempo completo en pregrado y posgrado en la Facultad de Odontología de la Universidad Autónoma de Nuevo León (UANL).

E-mail: ncfierrob@hotmail.com / norma.cruz.fr@uanl.edu.mx.

${ }^{3}$ Licenciada en Psicología. Maestría en Ciencias con Especialidad en Psicología de la Salud. Doctora en Metodología de las Ciencias del Comportamiento. Profesor de tiempo completo en pregrado y posgrado en la Facultad de Psicología (UANL).E-mail: monygzz77@yahoo.com.

${ }^{4}$ Licenciado en Psicología. Licenciado en Diseño Gráfico. Maestría en Arte Moderno y Contemporáneo. Doctor en Filosofía con Orientación en Psicología. Profesor de tiempo completo en pregrado y posgrado en la Facultad de Psicología y en la Facultad de Organización Deportiva (UANL). E-mail: minerva.vanegas@gmail.com.
\end{abstract}

Facultad de Odontología Dr. Eduardo Aguirre Pequeño, Universidad Autónoma de Nuevo León (UANL). - Monterrey, Nuevo León, México.

\section{Resumen}

El diagnóstico adecuado del bruxismo incluye el análisis y la correlación de signos y síntomas con diferentes métodos de diagnóstico, siendo esencial la interpretación y evaluación de los síntomas que reporta el paciente. El presente es un trabajo inicial sobre las propiedades psicométricas del cuestionario de bruxismo autoinformado (CBA). Participaron 100 personas (34 hombres y 66 mujeres) del noreste de México, la muestra se dividió en dos grupos, uno con diagnóstico clínico y autoinforme de bruxismo $(\mathrm{N}=50)$ y otro sin bruxismo. Se evaluó la confiablidad por consistencia interna, la estructura factorial y la validez convergente entre la escala de estrés percibido PSS-14 y el CBA. Los resultados muestran consistencia interna entre los 11 ítems $(\alpha=.88)$. El análisis factorial confirmatorio para un solo factor presentó índices estadísticos de bondad de ajuste adecuados $\left(\chi^{2} / \mathrm{gl}=1.461 ; \mathrm{GFI}=.916 ; \mathrm{AGFI}=.857\right.$, $\mathrm{CFI}=.967, \mathrm{RMSEA}=.068)$. La correlación entre las escalas PSS-14 y CBA fue positiva $(r=.27 ; p=.001)$, y entre la escala de estrés percibido y las subescalas bruxismo de sueño $r=.20(p=.039)$ y bruxismo de vigilia $r=.29$ $(p=.002)$, La correlación entre las subescalas BS y BV fue $r=.71(p=.001)$, Los resultados del CBA muestran alta confiabilidad, consistencia interna y correlación inter-ítems apropiada, y respaldan la validez convergente del cuestionario. Asimismo, contribuyen a 
mejorar los estándares de calidad al evaluar los datos subjetivos aportados por personas con bruxismo y se considera de interés continuar con el análisis del CBA, evaluando su comportamiento en otras poblaciones.

\section{Palabras clave: Bruxismo; Autoinforme;} Estrés percibido; Validez convergente; Análisis factorial.

\section{Abstract}

Bruxism is defined as a repetitive jaw-muscle activity, characterized by grinding or clenching the teeth, and /or by bracing or thrusting of the mandible, which has different circadian manifestations, either during sleep (sleep bruxism) or when wakefulness (awake bruxism). For an appropriate clinical diagnosis of bruxism different techniques have been proposed; the most common technique is the usage of a self-reported questionnaire combined with a professional evaluation. While the first is a questionnaire in which the patient expresses their symptoms, the second depends on the presence of observed signs associated according to an evaluator, including tooth wear as well as identify symptoms associated to this jaw-muscle activity, to confirm a clinical diagnosis, there must be a correlation between the self-report questionnaire and clinical diagnosis.

Therefore, there is no quantifiable measure that may help to evaluate, from a patient's perspective, the signs and symptoms that belongs to his/her muscle and mandibular activity while assessing both its proportion and measure. To strengthen the self-report technique as a reliable procedure for assessing the information expressed by patient with bruxism, this initial study reports the reliability and validity proprieties of the self-report bruxism questionnaire (CBA).

The present study was conducted in Mexico and includes one hundred participants. Fifty percent of them presented a clinical diagnosis of bruxism $(n=50)$, the other half (control group) do not. In both groups the perceived stress scale (PSS) and the self-reported bruxism questionnaires (CBA) were equally completed on a self-administered way.

Both a high internal consistency $(\alpha=.884)$ was found on the eleven items and a corrected item-total correlation ranged from .43 to .78 , reflects an adequate reliability.

The exploratory factor analysis was used to group the CBA variables; the extraction was made using a main component with Varimax rotation method. The sample adequacy coefficients, the Kaiser Meyer-Olkin $(\mathrm{KMO}=.826)$ and Bartlett's Sphericity test $\left(\chi^{2}=579.866\right.$, $p=.000)$ were assessed and expressed an appropriate intercorrelation between items. The factor analysis showed a bifactorial: the latent variables explained one factor of $47.8 \%$ (eigenvalue $=5.26)$ variance and another of $11.5 \%$ (eigenvalue $=1.27$ ), thus its cumulative variance of this model was $59.36 \%$.

Due to the amount of explained variance and the difference between the eigen values of this initial solution we considered to prove a unidimensional model. A one solution factor scale was feasible, with factor weights greater than .52 on all items. This singlefactor structure was verified in a confirmatory factor analysis, which presented significant values and suitable goodness of fit indexes $\left(\chi^{2} / \mathrm{df}=1.461 ; \mathrm{GFI}=.916 ; \mathrm{AGFI}=.857, \mathrm{CFI}=.967\right.$, RMSEA $=.068$ ). Also, a convergent analysis was performed proving a positive $(r=.27$; $p=.001)$ correlation between the PSS-14 and CBA scales, the correlation with the subscales of self-reported awake bruxism, SAB was positive $(r=.299, p=.002)$. Regarding self-reported sleep bruxism, SAB the correlation was also positive $(r=.207, p=.039)$. The correlation between the subscales was both positive and strong $(r=.713, p=.001)$.

Based on the results obtained, we can conclude that, there are a positive, significant, and adequate correlation between PSS-14 scale and the scale of self-reported bruxism, as though as in the sub-scales SAB and SSB. 
These results suggested that the one factor solution of the CBA has high reliability, internal consistency, and an appropriate inter-item correlation; also, it supports the convergent validity of the questionnaire. Due to the possible contributions of the CBA to the improvement of the quality standards of the evaluation of subjective data provided by people with bruxism, it is suggested to continue with the analysis of the scale in future studies with other populations.

Keywords: Bruxism; Self-report; Perceived stress; Convergent validity; Factorial analysis.

\section{Introducción}

La actividad repetitiva musculo-mandibular de apretar o rechinar los dientes, es un rasgo físico característico de las personas con bruxismo que puede presentarse en diferentes momentos del día, ya sea durante el sueño (bruxismo del sueño) o durante la vigilia (bruxismo despierto) (Lobbezoo et al., 2013).

Para su adecuado diagnóstico se han propuesto diferentes técnicas, una de las más comunes es el cuestionario de autoinforme, en el cual el paciente expresa sus síntomas. Estos cuestionarios generalmente deben complementarse con la evaluación clínica realizada por un odontólogo para identificar la presencia de signos y síntomas asociados a bruxismo; sin embargo, los datos que se obtienen en esta medición son aproximados porque varían según el criterio de quien lo mide (Paesani, 2010; Rintakoski, Hublin, Lobbezoo, Rose y Kaprio, 2012). Actualmente se ha establecido que para confirmar el diagnóstico clínico debe haber correlación entre el cuestionario de bruxismo autoinformado y el diagnóstico clínico realizado por un experto (de la Hoz, Díaz, LaTouche y Mesa, 2011; Lobbezoo et al., 2013; Paesani et al., 2013).

La literatura describe diferentes tipos de bruxismo, con procedimientos de diagnóstico específicos; en el caso del bruxismo durante el sueño se sugieren métodos diagnósticos como la electromiografía, la polisomnografía o el electroencefalograma. Dentro de los inconvenientes reportados para estas prácticas, están el alto costo y la incomodidad del paciente de dormir en un laboratorio conectado a aparatos, generando un ambiente fuera de lo común, circunstancias que podrían alterar el sueño del paciente y afectar el resultado de estas pruebas. Se ha usado también electromiografía portátil, sus ventajas son su costo accesible y que el registro de los datos lo hace el paciente en su casa; sin embargo, los datos que se registran pueden confundirse con otras actividades motoras como suspirar, toser o succionar (de la Hoz et al., 2011).

Relacionado con el diagnóstico y clasificación de bruxismo se han planteado diferentes criterios. Lobbezoo et al. (2013) propusieron la siguiente clasificación: (a) Posible bruxismo de vigilia o durante el sueño, basado en la confirmación de síntomas por medio de cuestionarios de autoinforme o por examen clínico. (b) Probable bruxismo de vigilia o durante el sueño, basado en el cuestionario junto con la evaluación por medio de examen clínico. (c) Definitivo bruxismo durante el sueño basado en el cuestionario de autoinforme, examen clínico y registros con electromiografía, o grabaciones de audio o video. El diagnóstico definitivo de bruxismo de vigilia lo basan en el cuestionario de autoinforme, examen clínico y grabación con electromiografía.

Por otra parte, Paesani $(2010,2013)$ establecieron que el diagnóstico se aproxima a probable bruxismo cuando se confirma la presencia de signos y síntomas de bruxismo ya sea por autoinforme, por entrevista o por el diagnóstico clínico realizado por un experto, y diagnóstico definitivo, al observar una alta correlación entre el auto-reporte y el diagnóstico clínico minucioso.

En los diferentes trabajos de investigación se observa que sin importar el método de diagnóstico que se siga, es frecuente el uso del autoinforme de los síntomas como un procedimiento de diagnóstico complemen- 
tario (Paesani et al., 2013). Por esta razón es fundamental tener un instrumento que evalúe cuantitativamente los signos y síntomas del paciente, para definir cuáles pertenecen específicamente a esta actividad musculo-mandibular, incluyendo la medida de su proporción. De esta forma el diseño del instrumento debe permitir explorar los puntos en común que tiene este grupo de pacientes y destacar la presencia de otras variables relacionadas, desde la perspectiva etiológica.

Para evaluar validez, los instrumentos bien diseñados generalmente comparan sus puntuaciones con escalas que miden el mismo constructo o relacionados. En esta investigación se obtuvo evidencia de validez convergente del cuestionario de bruxismo autoinformado, al correlacionarlo con el estrés psicológico. Esta variable fue seleccionada debido a que en informes anteriores se ha reportado la asociación del bruxismo a factores como ansiedad y estrés psicológico crónico, considerándolos como factores predisponentes, ya que el bruxismo es referido como un trastorno de la sensación, función y conducta, estrechamente relacionado con problemas o eventos estresantes (Abekura et al., 2011; American Academy of Sleep Medicine, 2005; Clasificación Internacional de Enfermedades, 2008).

Investigaciones recientes han evaluado estas variables psicológicas usando instrumentos ampliamente validados; sin embargo, son escasos los datos asociados a la confiabilidad, validez y datos normativos de algún cuestionario de bruxismo autoinformado. Por esta razón, en este trabajo se usó la escala de estrés percibido (PSS; Cohen, Kamarak y Mermelstein, 1983), en su versión adaptada para México por González y Landero (2007). Esta escala se basa en la teoría transaccional del estrés (Lazarus y Folkman, 1984) y evalúa si la persona se siente amenazada o afectada por situaciones, ya que cada persona responde de manera diferente a un estímulo común (García et al., 2011; González, 2011).

La base del Cuestionario de Bruxismo Autoinformado (CBA) es el autoinforme de los síntomas, ya que éstos son una referencia subjetiva que indica efectivamente la presencia de trastornos de salud. Mientras que la mayoría de los cuestionarios de bruxismo permiten opciones de respuesta dicotómicas sí / no, dando un nivel nominal o clasificación, el presente cuestionario tiene un diseño tipo Likert.

El estudio que se informa tiene como objetivo evaluar la confiabilidad y validez factorial y convergente del CBA.

\section{Método}

\section{Participantes}

El estudio se realizó en el noreste de México, se trabajó con una muestra no probabilística incidental. Participaron 34 hombres y 66 mujeres $(\mathrm{n}=100)$, con edades entre 14 y 69 años $(M=34.49$ años $)$, divididos en dos grupos, uno con diagnóstico clínico de bruxismo $(\mathrm{n}=50)$ y otro sin bruxismo $(n=50)$. Si bien la muestra es pequeña, puede considerarse adecuada para el análisis considerando el número de ítems del CBA y que se trata de un estudio inicial o piloto sobre las propiedades psicométricas del cuestionario.

Todos los participantes fueron contactados en diferentes centros dentales en el noreste de México. Para asegurar que los grupos y subgrupos estuvieran bien representados, todos los participantes se evaluaron usando el mismo procedimiento que incluyó: entrevista, examen clínico para diagnosticar signos o síntomas de bruxismo en dientes, periodonto, evaluación de los músculos masticatorios, cuello y / o parte superior de la espalda y la articulación temporomandibular. Este procedimiento se realizó conforme a los criterios diagnósticos de investigación de la guía clínica del trastorno temporomandibular (RDC) (TMD; Dworkin y LeResche, 1992), así como las recomendaciones de diferentes autores (Paesani et al., 2013; Rasit, Tutuncu y Acikel, 2012). Todos los participantes contestaron los cuestionarios PSS y CBA de forma auto-administrada, siendo voluntaria y anónima su participación. 


\section{Procedimiento}

Siguiendo los criterios de selección y el protocolo establecido, un odontólogo con experiencia realizó el diagnóstico clínico identificando la presencia de signos y síntomas reconocibles de bruxismo, e interrogó a cada paciente con el fin de hacer una interpretación adecuada y confirmar los datos. Para determinar si rechinaba los dientes en vigilia o durante el sueño, se identificó la presencia de áreas de desgaste en superficies oclusales o bordes incisales, los síntomas se confirmaron por el paciente o por referencia de una tercera persona. El criterio para confirmar si apretaba los dientes durante la vigilia o el sueño, fue por autoinforme del paciente y mostrar al menos dos de los siguientes signos o síntomas: músculo masetero con dolor o hipertónico, lesiones o línea alba en la mucosa bucal o lengua festoneada.

Como complemento de diagnóstico, cada participante recibió dos guardas termo conformados calibre .060, para uso exclusivo en vigilia o durante el sueño. Se evaluó la presencia de desgaste después de treinta días de uso, considerando que el uso del guarda no altera la calidad del sueño (Takahashi et al., 2013), ni inhibe la actividad del músculo masetero durante el sueño (Sjoholm et al., 2014).

De esta forma los criterios de selección para el grupo de personas con bruxismo fueron: el autoinforme de síntomas de bruxismo en vigilia o durante el sueño. Dichos síntomas y signos se confirmaron por medio de la entrevista, el diagnóstico clínico y el desgaste hecho a los guardas. En los criterios de selección para el grupo control o no bruxismo, se consideraron pacientes con dentadura completa, oclusión estable y sin evidencia de signos de bruxismo ni lesiones relacionadas.

Como criterios de exclusión en ambos grupos, se descartaron personas bajo tratamiento ortodóntico, que presentaran trastornos neurológicos, dolor crónico en otras partes del cuerpo, enfermedades reumáticas crónicas, que consumieran medicamentos para tratar cualquier enfermedad psicopatológica, que tomaran drogas que afecten tanto al sistema serotoninérgico o central, o por consumo recreativo 6 meses previos al estudio (de la Hoz et al., 2011; Paesani et al., 2013).

\section{Instrumentos}

Se usó la Escala de Estrés Percibido (PSS) de Cohen et al.(1983), adaptada a México por González y Landero (2007), para evaluar si el participante percibe su vida impredecible, sobrecargada e incontrolable. Esta escala tiene un formato tipo Likert de 14 ítems, con cinco posibles respuestas ( 0 : nunca a 4 : muy a menudo). Consta de 2 factores: el factor 1 , percepción positiva (ítems 4, 5, 6, 7, 9, 10, 13), y el factor 2, percepción negativa (ítems $1,2,3,8,11,12,14)$. La puntuación directa de la PSS-14 indica mayor nivel de estrés percibido con la puntuación más alta. El estrés bajo corresponde a una desviación estándar por debajo de la media y el estrés alto a una desviación estándar por encima de la media, tomando como referencia los puntajes del estudio de González, Rodríguez y Landero (2013). La confiabilidad de la versión adaptada es $\alpha=.84$. y el resultado en este trabajo muestra un coeficiente de confiabilidad adecuado, $\alpha=.79$ (ver Anexo 1).

El cuestionario de bruxismo autoinformado (CBA) se elaboró a partir de las preguntas utilizadas por diferentes autores (Abekura et al., 2011; de la Hoz et al., 2011; Dworkin y LeResche, 1992; Koyano et al., 2008; Lobbezoo et al., 2013; Manfredini et al., 2012; Paesani et al., 2013; Rintakoski et al., 2012; Winocur et al., 2011) (ver Anexo 2). Debido a que en forma individual estos cuestionarios contienen pocos ítems, se juntaron 11 preguntas revisando que el contenido incluyera lo necesario para evaluar el bruxismo autoinformado, y de esta forma contar con un instrumento de medición que permitiera obtener datos sobre la percepción del bruxismo en los participantes. El cuestionario propuesto es una escala tipo Likert con cinco posibles 
respuestas (1: nada a 5: mucho). La puntuación directa de la suma de los 11 ítems indica mayor percepción de bruxismo (ver Anexo 3).

\section{Análisis estadístico}

Se usó el software estadístico SPSS de IBM versión 21 (2012a), se obtuvo la media y desviación estándar de las variables, la confiabilidad de las escalas se verificó por medición de su consistencia interna. Se realizó el análisis factorial exploratorio siguiendo el método de componentes principales con rotación varimax. Para el análisis factorial confirmatorio, se utilizó el software AMOS versión 21 (IBM, 2012b). Considerando como valores adecuados los parámetros recomendados por Hooper, Coughlan y Mullen (2008), CMIN o Chi cuadrado entre los grados de libertad $\left(\chi^{2} / \mathrm{gl}\right)$ menor de 5 indica ajuste razonable; goodness-of-fit statistic (GFI) cercano a 1 se considera adecuado; adjusted goodness-of-fit statistic (AGFI) mayor que .9; Tucker-Lewisindex (TLI), normed-fit index (NFI) mayor que .9; comparative-fitindex (CFI) .95; standardized-root-mean squared-residual (SRMR) .08; root-mean-square-error of approximation (RMSEA) valor ideal menor a .05.
Se utilizó la correlación de Pearson para analizar la relación entre las variables. Para evaluar el mejor umbral para el cuestionario, se realizó una curva ROC (Receiver Operating Characteristics) junto con el valor AUC (Area Under the ROC Curve) por un método no paramétrico.

\section{Resultados}

La puntuación de la escala PSS, según los puntos de corte propuestos por González et al. (2013), indica que la muestra se encuentra en el nivel de estrés esperado, en relación con la edad y género (Tabla 1), es decir, el estrés no alcanzó puntuaciones para ser considerado alto o severo. Los puntajes de estrés percibido pueden oscilar entre 0 y 56 puntos, en este trabajo el puntaje promedio para la muestra total fue de 21.17 puntos, representando el $38.67 \%$ del máximo posible. En el grupo con diagnóstico clínico de bruxismo, el puntaje promedio fue .98 mayor que en el grupo sin bruxismo. En cuanto a las diferencias por género, la puntuación total de las mujeres fue 1.99 más alta para los pacientes en el grupo caso, que en el grupo control, a diferencia de los hombres en el grupo caso, donde la puntuación total fue 1.77 menor que en el grupo de control.

Tabla 1. Puntuación de la escala PSS y puntos de corte por género y edad.

Estrés Percibido (PSS)

\begin{tabular}{ccccc}
\hline & & $\begin{array}{c}\text { Diagnóstico clínico } \\
\text { de bruxismo }(\mathrm{n}=50)\end{array}$ & $\begin{array}{c}\text { No bruxismo } \\
(\mathrm{n}=50)\end{array}$ & $\begin{array}{c}\text { (González et al., } \\
2013)\end{array}$ \\
Género & $\mathrm{N}=100$ & $(\mathrm{n}=15) 19.93$ & $(\mathrm{n}=19) 21.10$ & $<13.38 ;>30.66$ \\
Hombre & $(\mathrm{n}=34) 20.58$ & $(\mathrm{n}=35) 22.40$ & $(\mathrm{n}=31) 20.41$ & $<15.28 ;>31.48$ \\
Mujer & $(\mathrm{n}=66) 21.46$ & $(\mathrm{n}=50) 21.66$ & $(\mathrm{n}=50) 20.68$ & $<15.95 ;>30.45$ \\
Total & $(\mathrm{n}=100) 21.17$ & & & \\
& & & & $<12.16 ;>29.70$ \\
Edad & & $(\mathrm{n}=17) 23.70$ & $(\mathrm{n}=15) 22.66$ & $<15.22 ;>31.48$ \\
$<24$ & $(\mathrm{n}=32) 23.21$ & $(\mathrm{n}=8) 25.00$ & $(\mathrm{n}=16) 18.12$ & $<13.85 ;>31.85$ \\
$25-34$ & $(\mathrm{n}=24) 20.41$ & $(\mathrm{n}=9) 18.55$ & $(\mathrm{n}=8) 20.50$ & $<17.45 ;>33.81$ \\
$35-44$ & $(\mathrm{n}=17) 19.47$ & $(\mathrm{n}=11) 18.36$ & $(\mathrm{n}=8) 21.00$ & \\
$45-54$ & $(\mathrm{n}=19) 19.47$ & $(\mathrm{n}=5) 22.20$ & $(\mathrm{n}=50) 200$ & \\
$55-70$ & $(\mathrm{n}=100) 22.87$ & $(\mathrm{n}=50) 21.66$ & & \\
Total & & & & \\
\hline
\end{tabular}

Puntos de corte 
Al analizar el estrés percibido por subgrupos de casos, según los distintos momentos circadianos de auto-reporte de síntomas (Tabla 2), se observó mayor puntuación en el subgrupo bruxismo combinado (23.24), en contraste con el subgrupo durante la vigilia (20.50) y durante el sueño (19.33).

Para los resultados de la escala CBA, las puntuaciones de los síntomas de bruxismo pueden oscilar entre 1 y 55. El promedio total fue de $20.46(D E=8.91)$, lo que representa el $37.2 \%$ del máximo posible. El grupo de autoinforme combinado obtuvo mayor puntuación en esta escala (30.84) en contraste con los subgrupos bruxismo despierto (23.68) y bruxismo durante el sueño (22.44).

Tabla 2. Resultados de la escala PSS y del CBA por género y por diferentes momentos circadianos.

Escala de Estrés Percibido (PSS)

\begin{tabular}{cccc}
\hline Momentos circadianos & $n$ & $M$ & $D E$ \\
\hline No Bruxismo & 50 & 20.68 & 5.89 \\
Durante el sueño (BS) & 9 & 19.33 & 4.44 \\
Vigilia (BV) & 16 & 20.50 & 8.31 \\
Ambos momentos (BC) & 25 & 23.24 & 6.86 \\
Total Bruxismo & 50 & 21.66 & 7.00 \\
\hline Puntuación del Cuestionario de Bruxismo Autoinformado & & \\
\hline Momentos circadianos & $n$ & & \\
\hline No Bruxismo & $n$ & $D E$ \\
\hline Durante el sueño (BS) & 50 & 13.88 & 2.95 \\
Vigilia (BV) & 9 & 22.44 & 6.04 \\
Ambos momentos (BC) & 16 & 23.68 & 6.05 \\
Total Bruxismo & 25 & 30.84 & 8.00 \\
\hline Género & 50 & 27.04 & 7.96 \\
\hline Hombre & & & \\
\hline Mujer & 34 & 18.94 & 8.26 \\
\hline
\end{tabular}

La confiabilidad se evaluó con el alfa de Cronbach, obteniéndose un coeficiente de.88, que sugiere que los 11 ítems tienen una consistencia interna adecuada. La correlación inter-ítem osciló de .43 a .78, considerándose adecuada por presentar valores superiores a .35 (Spector, 1992).

El análisis factorial exploratorio se realizó siguiendo el método de componentes principales con rotación varimax. Se estimaron los coeficientes de adecuación de la muestra, Kaiser Meyer-Olkin $(\mathrm{KMO}=.826)$ y el test de Esfericidad de Bartlett $\left(\chi^{2}=579.866\right.$, $p=.001)$, los cuales expresaron una intercorrelación apropiada entre ítems. El análisis muestra dos factores con valores propios mayores que 1 . Con los dos factores extraídos, la varianza acumulada fue de $59.36 \%$, una varianza explicada de $47.8 \%$ (autovalor $=5.26$ ) en el primer factor y $11.5 \%$ en el 
segundo factor (autovalor $=1.27$ ). Estos datos sugieren que una solución unifactorial sería adecuada.

Debido a la cantidad de varianza explicada en el primer factor, y la diferencia entre los valores propios del primer al segundo factor, se consideró probar un modelo unidimensional, obteniendo pesos factoriales mayores que .52 en todos los ítems.

Por medio del software AMOS versión 21 (IBM, 2012b), se analizó el modelo unidimensional de 11 ítems mediante análisis factorial para estimar el efecto y las relaciones entre los ítems. El primer modelo (sin correlaciones) no mostró adecuados estadísticos de bondad de ajuste (Tabla 3); sin embargo, todos los parámetros (efectos de los ítems en la variable latente) fueron significativos, por lo que se usaron los índices de modificación para identificar los posibles pesos de regresión y covarianzas que pueden ser incorporados en un modelo re-especificado. Las correlaciones incluidas en el último modelo son teóricamente consistentes debido a la relación que hay entre los síntomas según la manifestación circadiana.

Este modelo muestra valores adecuados, en la tabla 3 se presentan las estadísticas de bondad de ajuste obtenidos en el modelo final con correlaciones (modelo 2 en la Tabla 3). Todos los parámetros son estadísticamente significativos. Los porcentajes de la varianza explicada de los ítems varían entre 26 y $71 \%$ y la parsimonia (PR.600).

Tabla 3. Análisis factorial confirmatorio del Cuestionario de Bruxismo Autoinformado, estimados estandarizados de los modelos de análisis estadístico, con criterios de bondad de ajuste.

\begin{tabular}{ccccccccccccc}
\hline Modelo & $\chi^{2}$ & $\mathrm{gl}$ & $\mathrm{p}$ & $\chi^{2} / \mathrm{gl}$ & GFI & AGFI & TLI & NFI & RMR & RMSEA & CFI & SRMR \\
\hline 1 & 190.966 & 44 & .000 & 4.340 & .764 & .646 & .667 & .686 & .133 & .184 & .734 & .0924 \\
2 & 34.34 & & .4 & 1.04 & .942 & .885 & .996 & .943 & .056 & .020 & .998 & .0403 \\
$\begin{array}{c}\text { Criterios } \\
\text { de }\end{array}$ & & & & & & & & & & & & \\
$\begin{array}{c}\text { bondad } \\
\text { de ajuste }\end{array}$ & & & & $<5$ & 1 & $>.9$ & $>.9$ & $>.9$ & $1 \mathrm{a} 7$ & $<.05$ & .95 & .08 \\
\hline
\end{tabular}

La Tabla 4 muestra las puntuaciones del cuestionario de bruxismo autoinformado. Sobre la base de estos datos, se propone que con puntajes de 0 a 18 es improbable que el paciente tenga síntomas de bruxismo, puntuaciones de 19 a 24 indicarían un puntaje promedio de diagnóstico clínico (probables síntomas de bruxismo) y una puntuación de 25 o superior sería indicativo de un diagnóstico de síntomas definitivos de bruxismo. 
Tabla 4. Percentiles y puntos de corte del Cuestionario de Bruxismo Autoinformado (CBA).

Diagnóstico clínico

\begin{tabular}{|c|c|c|c|c|}
\hline \multirow{12}{*}{ No bruxismo } & Medi & & 13.88 & \multirow{12}{*}{$\begin{array}{c}\text { Improbable } \\
\text { bruxismo }\end{array}$} \\
\hline & Media & & 13.50 & \\
\hline & \multicolumn{2}{|c|}{$\begin{array}{c}\text { Mediana } \\
\text { Desviación estándar }\end{array}$} & 2.95 & \\
\hline & \multirow{9}{*}{ Percentiles } & 10 & 11.0 & \\
\hline & & 20 & 11.0 & \\
\hline & & 30 & 12.0 & \\
\hline & & 40 & 13.0 & \\
\hline & & 50 & 13.5 & \\
\hline & & 60 & 14.0 & \\
\hline & & 70 & 14.7 & \\
\hline & & 80 & 15.0 & \\
\hline & & 90 & 18.0 & \\
\hline \multirow{7}{*}{$\begin{array}{l}\text { Definitivo } \\
\text { diagnóstico } \\
\text { clínico de } \\
\text { bruxismo }\end{array}$} & \multicolumn{2}{|c|}{ Media } & 27.04 & \multirow{12}{*}{ Probable bruxismo } \\
\hline & Media & & 25.00 & \\
\hline & Desviación & & 7.97 & \\
\hline & \multirow{9}{*}{ Percentiles } & 10 & 19.0 & \\
\hline & & 20 & 20.0 & \\
\hline & & 30 & 22.0 & \\
\hline & & 40 & 24.0 & \\
\hline \multirow{5}{*}{$\mathrm{n}=50$} & & 50 & 25.0 & \\
\hline & & 60 & 28.2 & \\
\hline & & 70 & 30.7 & \\
\hline & & 80 & 33.0 & \\
\hline & & 90 & 40.0 & \\
\hline
\end{tabular}

La correlación del CBA con los niveles de estrés fue positiva $(r=27, p=.006)$. Por lo tanto, cuanto mayor sea la puntuación de estrés percibido, mayor será la puntuación en los síntomas de bruxismo autoinformados. Además, la correlación con las subescalas de bruxismo autoinformado, en vigilia fue positiva $(r=.29, p=.002)$ y durante el sueño también $(r=.20, p=.039)$. La correlación entre las subescalas del CBA fue positiva $\mathrm{y}$ fuerte $(r=.71, p=.001)$.

Para evaluar el mejor umbral para el cuestionario, se realizó una curva ROC junto con el valor del AUC $(\mathrm{AUC}=.963, D E=.17 ; 95$ \% intervalo de confianza: .930-.997, $p<.001$ ). Esta curva y el AUC correspondiente muestran que el CBA tiene capacidad predictiva
Puntuación CBA

para discriminar el estado real positivo y el grupo de estado real negativo. El área bajo la curva ROC es de .05 ( $p=.0001)$, y muestra la capacidad del CBA, para distinguir entre los dos grupos (Zhou, Obuchowski y McClish, 2011).

\section{Discusión}

Se han realizado diferentes propuestas para estandarizar los métodos y criterios diagnósticos del bruxismo; sin embargo, cualquier método diagnóstico que se utilice es acompañado por el diagnóstico clínico y la interpretación de los signos y síntomas relacionados con la actividad musculo-mandibular que informa el paciente. 
Los cuestionarios de autoinforme de síntomas del bruxismo son un método de diagnóstico ampliamente utilizado por los clínicos e investigadores, ya que proporciona datos de las respuestas del paciente; no obstante, son instrumentos característicamente breves, como el propuesto por Ahlberg et al. (2013), que incluyen una sola pregunta para evaluar la frecuencia de apretar o rechinar los dientes, con opciones de respuesta: nunca, rara vez, a veces, a menudo y continuamente. O el de Rintakoski et al. (2012), en el que se le pregunta “¿Rechina sus dientes?”, con opciones de respuesta: "todas las noches", "semanalmente", "ocasionalmente (o menos frecuente que cada semana)", "nunca” y "no sé".

En contraste con las características que tienen los cuestionarios de ser breves, o en la mayoría de los casos incluyen respuestas dicotomizadas, una de las ventajas del CBA es la cantidad de ítems que se utilizan para evaluar la variedad de síntomas y opciones relacionadas con la frecuencia y duración (ya sea en vigilia o durante el sueño o ambos momentos), no solo para fines clínicos sino también para fines de investigación. Así, la diferencia con el cuestionario de bruxismo autoinformado (CBA), propuesto en el presente trabajo, es que mide los principales síntomas del bruxismo en una escala de tipo Likert con cinco opciones de respuesta (desde 1: nada, hasta 5: mucho), permitiendo establecer una gama de intensidad de percepción de estos síntomas, y al mismo tiempo, una correlación de estos rasgos de bruxismo con escalas psicológicas, como la escala de estrés percibido (PSS).

En estudios similares, como el de Winocur et al. (2011), se han reportado valores superiores en la escala PSS. En dicho estudio se evaluaron 402 adultos, y no se encontraron diferencias significativas $(p>.05)$ en el nivel de estrés entre el grupo de bruxismo de vigilia $(n=126, M=25.76, D E=6.61)$ y el grupo $\sin$ bruxismo $(n=275, M=24.49, D E=6.85)$. $\mathrm{Sin}$ embargo, los datos en relación con el género muestran diferencias significativas, con un promedio en hombres $(n=151)$ de 23.33 (DE $=7.57)$ y mujeres $(n=251)$, de $25.77(D E=$ 6.17) $(p<.001)$. Estos puntajes están dentro de lo esperado según lo propuesto por González et al. (2013) y coinciden con los resultados del presente estudio (Tabla 1).

Si bien el estrés es una de las variables más estudiadas asociadas al bruxismo, ya que es común que durante la entrevista el paciente atribuya el aumento de la actividad de apretar o rechinar sus dientes al estrés, a situaciones estresantes, a problemas en su vida, entre otras, es de interés mencionar que la correlación entre estrés percibido y el bruxismo autoinformado fue positiva. Los resultados obtenidos reflejan que, a mayor puntuación de estrés percibido, mayor será la puntuación en los síntomas de bruxismo autoinformados.

Sin embargo, debido a que el instrumento PSS analiza situaciones experimentadas dentro del último mes, se recomienda incluir preguntas que sitúen al paciente en los momentos o contextos de estrés agudo que incrementen sus síntomas de bruxismo.

Los resultados de la percepción de estrés en personas con bruxismo, reportados por diferentes autores, así como los resultados obtenidos en este estudio, muestran un nivel de estrés percibido similar al reportado por el grupo sin bruxismo, exponiendo su origen multifactorial, por lo que será importante realizar estudios más profundos de las variables psicológicas relacionadas con el proceso de estrés-emoción-manejo, con la finalidad de evaluar no solo su experiencia percibida ante un estresor, sino también su respuesta frente a las demandas sociales o psicológicas, y el grado y duración de las emociones negativas asociadas, ya que al prolongarse contribuirían a alterar el bienestar de las personas, así como también la capacidad de regulación emocional y su relación con la salud física (SamperGarcía, Mesurado, Richaud y Llorca, 2016).

Los resultados de validez y confiabilidad indican que el cuestionario de bruxismo autoinformado puede ser una opción factible para 
obtener información confiable en relación con los síntomas y el diagnóstico clínico del bruxismo, teniendo como referencia los puntajes propuestos en la Tabla 4. Sin excepción, los síntomas de bruxismo en vigilia y durante el sueño muestran una fuerte correlación entre los ítems, dando lugar a la solución univariante como la mejor estructura factorial para la escala, recomendando utilizar estos ítems para el autoinforme de síntomas de bruxismo de vigilia y durante el sueño, solo en la práctica clínica para tener detalles sobre el caso.

Dentro de las limitaciones del estudio está la validez externa, que representa la posibilidad de generalizar los resultados. La naturaleza no probabilística de esta muestra nos lleva a considerar los resultados de este estudio como datos iniciales, y sugerimos someter la escala a más estudios de validez y confiabilidad. Se recomienda además analizar la estabilidad temporal, por la confiabilidad del test-retest.

Al ser un estudio preliminar, se recomienda realizar investigaciones que incluyan grupos con diferentes trastornos temporomandibulares, cuyos síntomas pueden parecerse a los del bruxismo, a efectos de evaluar la capacidad discriminativa del cuestionario.

\section{Conclusión}

El objetivo de este estudio fue proporcionar evidencia de la estructura factorial del cuestionario de bruxismo autoinformado, así como la validez convergente y datos de confiabilidad de este cuestionario. Con base en los resultados obtenidos, podemos concluir que la confiabilidad de la escala de 11 ítems es aceptable, y que los mismos tienen una consistencia interna relativamente alta, la correlación corregida del ítem-total refleja valores de rango apropiados. La estructura de un solo factor sugerida por el análisis de componentes principales se verificó con el análisis factorial confirmatorio, en el cual todos los parámetros fueron significativos, y con adecuados índices de bondad de ajuste, permitiendo covarianza entre algunos errores de medida.
Existe una correlación positiva y significativa entre la escala de PSS-14 y el CBA, así como en las subescalas del autoinforme de bruxismo en vigilia y durante el sueño. Si bien la correlación entre las subescalas fue significativa, positiva y fuerte, se esperaba que los participantes presentaran puntuaciones altas de estrés; por el contrario, el nivel de estrés percibido de la muestra está dentro del rango esperado. La puntuación fue más alta en el grupo con bruxismo en contraste con el de no bruxismo, así como en el subgrupo con autoinforme de síntomas en ambos momentos (combinado vigilia y durante el sueño).

Se recomienda el uso del CBA como parte del registro clínico odontológico, así como incluirlo en la práctica como parte de los formatos que los pacientes responden antes de la consulta, ya que cuando el problema no ha alcanzado un daño significativo, algunos pacientes no lo consideran un problema, y no lo informan a su dentista, y pueden pasar años antes de iniciar un tratamiento. Los resultados de este estudio sugieren que el cuestionario es una herramienta factible para ser utilizado como screening.

\section{Referencias bibliográficas}

Abekura, H., Tsuboi, M., Okura, T., Kagawa, K., Sadamori, S. y Akagawa, Y. (2011). Association between sleep bruxism and stress sensitivity in an experimental psychological stress task. Biomedical Research, 32(6), 395-399. doi: 10.2220/biomedres.32.395.

Ahlberg, J., Lobbezoo, F., Ahlberg, K., Manfredini, D., Hublin, C, Sinisalo, J., Könönen, M. y Savolainen, A. (2013) Self-reported bruxism mirrors anxiety and stress in adults. Medicina Oral Patología Oral Cirugía.Bucal, 18(1), 7-11. doi: 10.4317/medoral.18232

American Academy of Sleep Medicine. (2005). Publication Diagnostic and coding manual. ICSD-2. International classification of sleep disorders Westchester. Illinois: Library of Congress. Recuperado de http://www.esst.org/ adds/ICSD.pdf 
Clasificación Internacional de Enfermedades (2008). Clasificación de los trastornos mentales y del comportamiento CIE-10 (pp. 136). México: Panamericana.

Cohen, S., Kamarck, T. y Mermelstein, R. (1983). A global measure of perceived stress. Journal of Health and Social Behavior, 24(4), 385-396. Recuperado de http://www.psy.cmu.edu/ scohen/globalmeas83.pdf

de la, Hoz, J., Díaz, E., La Touche, R. y Mesa, J. (2011). Sleep bruxism. Conceptual review and update. Revista Medicina Oral Patología Oral y Cirugía Bucal, 16(2), 231-238. doi: 10.4317/ medoral.16. e231

Dworkin, S. y LeResche, L. (1992). Research diagnostic criteria for temporomandibular disorders: review, criteria, examinations and specifications, critique. Journal of Craniomandibular Disorders, 6(4), 301-355.

García, G., Servera, M., Chellew, K., Meisel, V., Fornes, J., Cardo, E., Pérez, G., Riesco, M. y Ronald, M. (2011). Prosocial personality traits and adaptation to stress. Social Behavior and Personality, 39(10), 1337-1348. doi: 10.2224/ sbp.2011.39.10.1337

González, M. (2011). Síntomas psicosomáticos y estrés. En Sintomas psicosomáticos y estrés ¿cómo se relacionan? (pp. 30-40) México: IMJUVE.

González, M. y Landero, R. (2007). Factor structure of the perceived stress scale (PPS) in a sample from Mexico. The Spanish Journal of Psychology, 10(1), 199-206.

González, M., Rodríguez, A. y Landero, R. (2013). The Perceived Stress Scale (PSS): Normative data and factor structure for a largescale sample in Mexico. The Spanish Journal of Psychology, 16(5), 47-55. doi: 10.1017/ sjp.2013.35.

Hooper, D., Coughlan, J. y Mullen, M. (2008). Structural equation modelling: guidelines for determining model fit. The Electronic Journal of Business Research Methods, 6(1), 53-60.

IBM (2012a). SPSS Statistics 21.0.0. [Software]. Recuperado de

https://www-01.ibm.com/software/mx/ analytics spss/products/statistics/
IBM (2012b). SPSS AMOS 21.0.0 [Software]. Recuperado de http://www.spss.com.hk/amos/ index.htm?tab=1

Koyano, K., Tsukiyama, Y., Ichiki, R. y Kuwata, T. (2008). Review article assessment of bruxism in the clinic. Journal of Oral Rehabilitation, 35(7), 495-508. doi: 10.1111/j.13652842.2008.01880.x

Lazarus, R.S. y Folkman, S. (1984). Concept of coping. En Stress, coping and adaptation (pp. 306-364). Nueva York: Springer Publishing.

Lobbezoo, F., Ahlberg, J., Glaros, G., Kato, T., Koyano, K., Lavigne, G., Leeuw, R., Manfredini, D., Svensson, P. y Winocur, E. (2013). Bruxism defined and graded: an international consensus. Journal of Oral Rehabilitation, 40(1), 2-4. doi: 10.1111/joor. 12011

Manfredini, D., Winocur, E., Guarda, L. y Lobbezoo, F. (2012.). Self-reported bruxism and temporomandibular disorders: findings from two specialized centers. Journal of Oral Rehabilitation, 39(5), 319-325. doi: 10.1111/j.1365-2842.2011.02281.x

Paesani, D. (2010). Bruxism: Theory and practice. Reino Unido: Quintessence Publishing.

Paesani, D., Lobbezoo, F., Gelos, C., Guarda, L., Ahlberg, J. y Manfredini, D. (2013). Correlation between self-reported and clinically based diagnoses of bruxism in temporomandibular disorders patients. Journal of Oral Rehabilitation, 40(11), 803-809. doi: 10.1111/joor.12101

Rasit, G., Tutuncu, R. y Acikel, C. (2012). Psychopathological profile of patients with different forms of bruxism. Clinical Oral Investigation, 16(1), 305-311. doi: 10.1007/s00784-0100492-9

Rintakoski, K., Hublin, Ch., Lobbezoo, F., Rose, R. y Kaprio, J. (2012). Genetic factors account for half of the phenotypic variance in liability to sleep-related bruxism in young adults: a nationwide twin cohort study. Twin Research and Human Genetics, 15(6), 714-719. doi: 10.1017/thg. 2012.54

Samper-García, P., Mesurado, B., Richaud, M. y Llorca, A. (2016). Validación del cuestionario de conciencia emocional en adolescentes españoles. Interdisciplinaria, 33(1), 
163-176. Recuperado de http://www.redalyc. org/pdf/180/18049204010.pdf doi: 10.16888/ interd.2016.33.1.10

Sjoholm, T., Kauko, T., Kemppainen, P. y Rauhala, E. (2014). Long-term use of occlusal appliance has impact on sleep structure. Journal of Oral Rehabilitation, 41(6), 644- 652. doi: 10.1111/ joor. 12201

Spector, P. (1992). Conducting the item analysis. En Summated rating scale construction. An Introduction (pp. 29-46). California: Sage Publications.

Takahashi, H., Masaki, C., Makino, M., Yoshida, M., Mukaibo, T., Kondo, Y., Nakamoto, T. y
Hosokawa, R. (2013). Management of sleeptime masticatory muscle activity using stabilization splints affects psychological stress. Journal of Oral Rehabilitation, 40(12), 892-899. doi: 10.1111/joor.12110

Winocur, E., Uziel, N., Lisha, T., Goldsmith, C. y Eli, I. (2011). Self-reported bruxism associations with perceived stress, motivation for control, dental anxiety and gagging. Journal of Oral Rehabilitation, 38(1), 3-11. doi: 10.1111/j.13652842.2010.02118

Zhou, X., Obuchowski, N. y McClish, D. (2011). Statistical methods in diagnostic medicine. Estados Unidos: Wiley \& Sons, Inc. 


\section{ANEXO 1}

ESCALA DE ESTRES PERCIBIDO (PSS), versión en español adaptada para México (González y Landero, 2007).

Marca la opción que mejor se adecúe a tu situación actual, teniendo en cuenta el último mes.

\begin{tabular}{|c|c|c|c|c|c|}
\hline $\begin{array}{l}\text { 1. ¿Con qué frecuencia has estado afectado/a por algo que ha ocurrido } \\
\text { inesperadamente? }\end{array}$ & 0 & 1 & 2 & 3 & 4 \\
\hline $\begin{array}{l}\text { 2. ¿Con qué frecuencia te has sentido incapaz de controlar las cosas } \\
\text { importantes de tu vida? }\end{array}$ & 0 & 1 & 2 & 3 & 4 \\
\hline $\begin{array}{l}\text { 3. ¿Con qué frecuencia te has sentido nervioso/a o estresado/a (lleno/a de } \\
\text { tensión)? }\end{array}$ & 0 & 1 & 2 & 3 & 4 \\
\hline $\begin{array}{c}\text { 4. ¿Con qué frecuencia has manejado con éxito los pequeños problemas } \\
\text { irritantes de la vida? }\end{array}$ & 0 & 1 & 2 & 3 & 4 \\
\hline $\begin{array}{l}\text { 5. ¿Con qué frecuencia has sentido que hayas enfrentado efectivamente } \\
\text { los cambios importantes que han estado ocurriendo en tu vida? }\end{array}$ & 0 & 1 & 2 & 3 & 4 \\
\hline $\begin{array}{l}\text { 6. ¿Con qué frecuencia has estado seguro/a sobre tu capacidad de manejar } \\
\text { tus problemas personales? }\end{array}$ & 0 & 1 & 2 & 3 & 4 \\
\hline 7. ¿Con qué frecuencia has sentido que las cosas te salen bien? & 0 & 1 & 2 & 3 & 4 \\
\hline $\begin{array}{l}\text { 8. ¿Con qué frecuencia has sentido que no podías enfrentar todas las cosas } \\
\text { que tenías que hacer? }\end{array}$ & 0 & 1 & 2 & 3 & 4 \\
\hline 9. ¿Con qué frecuencia has podido controlar las dificultades de tu vida? & 0 & 1 & 2 & 3 & 4 \\
\hline 10. ¿Con qué frecuencia has sentido que tienes el control de todo? & 0 & 1 & 2 & 3 & 4 \\
\hline $\begin{array}{l}\text { 11. ¿Con qué frecuencia has estado enojado/a porque las cosas que te han } \\
\text { ocurrido estaban fuera de tu control? }\end{array}$ & 0 & 1 & 2 & 3 & 4 \\
\hline $\begin{array}{l}\text { 12. ¿Con qué frecuencia has pensado sobre las cosas que no has terminado } \\
\text { (pendientes de hacer)? }\end{array}$ & 0 & 1 & 2 & 3 & 4 \\
\hline $\begin{array}{l}\text { 13. ¿Con qué frecuencia has podido controlar la forma de pasar el tiempo } \\
\text { (organizar)? }\end{array}$ & 0 & 1 & 2 & 3 & 4 \\
\hline $\begin{array}{l}\text { 14. ¿Con qué frecuencia has sentido que las dificultades se acumulan tanto } \\
\text { que no puedes superarlas? }\end{array}$ & 0 & 1 & 2 & 3 & 4 \\
\hline
\end{tabular}


ANEXO 2

Frecuencia de las preguntas incluidas en diferentes publicaciones por autor y año.

$\begin{array}{cccccccccc} & \text { RDC- } & \text { Koyano } & \text { Paesani } & \text { Abekura } & \text { Hoz de } & \text { Winocur } & \text { Manfredini } & \text { Rintakoski } & \text { Lobbezoo } \\ \text { Pregunta } & \text { TMD } & \text { et al. } & (2010) & \text { et al. } & \text { la et al. } & \text { et al. } & \text { et al. }(2012) & (2012) & (2013)\end{array}$

\begin{tabular}{|c|c|c|c|c|c|c|c|c|c|}
\hline $\begin{array}{c}\text { 1. ¿Se ha dado cuenta que } \\
\text { rechina o aprieta los dientes } \\
\text { con frecuencia durante el } \\
\text { sueño? }\end{array}$ & $\mathrm{x}$ & & $x$ & $x$ & $x$ & & $x$ & & $\mathrm{x}$ \\
\hline $\begin{array}{l}\text { 2. ¿Alguien lo ha } \\
\text { escuchado rechinar los } \\
\text { dientes por la noche? }\end{array}$ & & $x$ & $x$ & & $x$ & $x$ & & & \\
\hline $\begin{array}{c}\text { 3. ¿Ha sentido su } \\
\text { mandíbula fatigada o con } \\
\text { dolor al despertar por la } \\
\text { mañana? } \\
\end{array}$ & $x$ & $x$ & $x$ & $x$ & $x$ & $x$ & & & \\
\hline $\begin{array}{l}\text { 4. ¿Alguna vez siente dolor } \\
\text { momentáneo de cabeza al } \\
\text { despertar por la mañana? }\end{array}$ & $x$ & $x$ & $x$ & $x$ & $x$ & $x$ & & & \\
\hline $\begin{array}{l}\text { 5. ¿Ha notado que rechina } \\
\text { los dientes durante el día? }\end{array}$ & $\mathrm{x}$ & $x$ & $x$ & & & & $x$ & $x$ & $x$ \\
\hline $\begin{array}{l}\text { 6. ¿Presenta dificultad } \\
\text { para abrir bien la boca al } \\
\text { despertar? }\end{array}$ & $x$ & $x$ & & $x$ & $x$ & $x$ & & & \\
\hline $\begin{array}{c}\text { 7. ¿Se ha dado cuenta } \\
\text { de que presenta desgaste } \\
\text { considerable en sus dientes? }\end{array}$ & & $\mathrm{x}$ & & $\mathrm{x}$ & $x$ & $x$ & & & $\mathrm{X}$ \\
\hline $\begin{array}{l}\text { 8. ¿Siente dolor en sus } \\
\text { dientes al contacto con el } \\
\text { aire o líquidos fríos? }\end{array}$ & & $\mathrm{x}$ & & & $x$ & $x$ & & & \\
\hline $\begin{array}{l}\text { 9. ¿Ha notado que aprieta } \\
\text { los dientes durante el día? }\end{array}$ & $x$ & $x$ & $x$ & & & & $\mathrm{X}$ & & $x$ \\
\hline $\begin{array}{c}\text { 10. ¿Siente que se le } \\
\text { traba la articulación de } \\
\text { la mandíbula o que hace } \\
\text { un sonido como clic al } \\
\text { moverla? }\end{array}$ & $x$ & $x$ & $x$ & & $x$ & $x$ & & & \\
\hline $\begin{array}{l}\text { 11. ¿Siente sus dientes } \\
\text { o encías con dolor al } \\
\text { despertar por la mañana? }\end{array}$ & & $x$ & $x$ & & $\mathrm{X}$ & & & & \\
\hline
\end{tabular}

Nota: Se realizó la traducción de las preguntas para este estudio 


\section{ANEXO 3}

CUESTIONARIO DE BRUXISMO AUTOINFORMADO (CBA)

Instrucciones: A continuación, se le presentan una serie de preguntas relacionadas con su actividad dental/bucal. Por favor conteste en las columnas de la derecha la respuesta que mejor represente el estado actual de dicha situación en su vida durante los últimos seis meses.

\begin{tabular}{|c|c|c|c|c|c|c|}
\hline & Preguntas & $\begin{array}{l}\frac{\pi}{\tilde{J}} \\
\text { Z } \\
-\end{array}$ & $\begin{array}{l}8 \\
8 \\
0 \\
\text { ¿. }\end{array}$ & 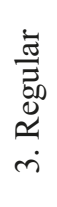 & $\begin{array}{l}\frac{0}{\pi} \\
\frac{\pi}{0} \\
\frac{0}{0} \\
\dot{\Sigma} \\
\dot{\nabla}\end{array}$ & $\underset{\dot{i}}{\stackrel{0}{0}}$ \\
\hline 1. & $\begin{array}{c}\text { ¿Se ha dado cuenta que rechina o aprieta los dientes con } \\
\text { frecuencia durante el sueño? }\end{array}$ & & & & & \\
\hline 2. & ¿Alguien lo ha escuchado rechinar los dientes por la noche? & & & & & \\
\hline 3. & $\begin{array}{l}\text { ¿Ha sentido su mandíbula fatiga o con dolor al despertar por la } \\
\text { mañana? }\end{array}$ & & & & & \\
\hline 4. & $\begin{array}{l}\text { ¿Alguna vez siente dolor momentáneo de cabeza al despertar } \\
\text { por la mañana? }\end{array}$ & & & & & \\
\hline 5. & ¿Ha notado que rechina los dientes durante el día? & & & & & \\
\hline 6. & ¿Presenta dificultad para abrir bien la boca al despertar? & & & & & \\
\hline 7. & $\begin{array}{l}\text { ¿se ha dado cuenta que presenta desgaste considerable en sus } \\
\text { dientes? }\end{array}$ & & & & & \\
\hline 8. & $\begin{array}{c}\text { ¿Siente dolor en sus dientes al contacto con el aire o líquidos } \\
\text { fríos? }\end{array}$ & & & & & \\
\hline 9. & ¿Ha notado que aprieta los dientes durante el día? & & & & & \\
\hline 10. & $\begin{array}{c}\text { ¿Siente que se le traba la articulación de la mandíbula o que } \\
\text { hace un sonido como clic al moverla? }\end{array}$ & & & & & \\
\hline 11. & $\begin{array}{c}\text { ¿Siente sus dientes o encías con dolor al despertar por la } \\
\text { mañana? }\end{array}$ & & & & & \\
\hline
\end{tabular}

Recibido: 24 de diciembre de 2017 Aceptado: 25 de septiembre de 2019 